\title{
MONOMIAL CURVES AND OBSTRUCTIONS \\ ON CYCLIC QUOTIENT SINGULARITIES
}

\author{
Jan Arthur Christophersen \\ Institute of Mathematics \\ University of Oslo \\ Oslo, Norway
}

\section{Introduction}

We show how the obstruction space $\mathrm{T}^{2}$ for isolated singularities is related to the deformations of certain hypersurface sections $(\S 1)$. This is applied to 2 dimensional cyclic quotient singularities, or what is the same thing; 2 dimensional normal affine toric varieties. For more on quotient singularities see e.g. $[B r],[B-K-R]$, for toric varieties see, e.g. $[K-K-M-S]$. We find a monomial curve $C$ on the cyclic quotient $X$ such that

$$
\tau_{C}-\mu_{C}=\operatorname{dim}_{C_{X}}^{2}+t_{C}-1
$$

Here $\tau$ is the Tjurina number, $\mu$ is the Milnor number and $t$ is the Gorenstein type. $\tau$ is computed using a method that works for all affine toric varieties $(\$ 2)$ and $\mu$ by a Kouchnirenko type formula for functions on cyclic quotient surfaces, (\$4).

If $r$ is the minimal codimension of $x, i \cdot e \cdot r=d i m_{C}-m_{, 0} / m_{X}^{2}, 0-2$, then

$$
\operatorname{dim}_{\mathbb{C}^{\mathrm{T}}} \mathrm{X}^{2}=r(r-2)
$$

when $r \geqslant 2$. (Of course $T_{X}^{2}$ is trivial when $r=1$ ). I have learned through private communication that Jürgen Arndt (Hamburg) has computed this dimension by a different method.

I would like to thank Olav Arnfinn Laudal for constant help and advice. I have also benefited from conversations with A. Sletsjфe, 
J. Damon, G.-M. Greuel and the stimulating milieu at Lambrecht. (Supported by the Norwegian Research Council for Science and the Humanities.)

\section{Obstructions and deformations of hypersurface sections}

Throughout we will work over the ground field $\mathbb{C}$. We use the notation $\mathrm{T}_{X}^{i}$ for $\mathrm{T}^{\mathrm{i}}\left(\mathrm{X} / \boldsymbol{C}, \mathrm{O}_{\mathrm{X}}\right.$ ) (cotangent complex notation, see $[\mathrm{Li}-\mathrm{S}]$ ) and $\mathrm{H}^{\mathrm{i}}\left(\boldsymbol{C}, \mathrm{X} ; \mathrm{O}_{\mathrm{X}}\right.$ ) (André cohomology notation, see $[L]) . T_{X}^{1}$ is the space classifying infinitesimal deformations; the tangent space of the miniversal deformation space. $\mathrm{T}_{\mathrm{X}}^{2}$ is the space "in which the obstructions lie". (See $[L],[R],[S]$ for the deformation theory involved.)

Lemma 1.1. Let $\mathrm{X}$ be an affine scheme with one singular point $\mathrm{x}$ and $f \in \underline{m}_{x}, x^{\prime}$ the maximal ideal, such that

(i) $f: X \rightarrow \mathbb{C}$ has one critical point $x$, and $f(x)=0$.

(ii) $f \in \operatorname{Ann}\left(T_{X}^{2}\right)$.

If $Y=f^{-1}(0)$ then

$$
\operatorname{dim}_{\mathbb{C}} T_{Y}^{1}-e_{Y}=\operatorname{dim}_{\mathbb{C}} T_{X}^{2}
$$

where $e_{Y}$ is the dimension of a smoothing component of the versal deformation space of $Y$.

By a smoothing we mean a deformation with smooth generic fiber. Thus over a smoothing component the generic fiber is non-singular.

Proof. This is a corollary of "Wahl's conjecture" on the dimension of smoothing components recently proved in $[G-L]$ and $[L-P]$.

Obviously $f: X \rightarrow \mathbb{C}$ is a smoothing of $Y$. From the exact sequerce

$$
0 \rightarrow \mathbb{C}[t] \stackrel{+t}{\rightarrow} \mathbb{C}[t] \rightarrow \mathbb{C} \rightarrow 0
$$

we get a long exact sequence in algebra cohomology 


$$
\begin{aligned}
& O \rightarrow \mathrm{H}^{0}\left(\mathbb{C}[t], \mathrm{X} ; \mathrm{O}_{\mathrm{X}}\right) \cdot \mathrm{H}^{0}\left(\mathbb{C}[t], \mathrm{X} ; \mathrm{O}_{\mathrm{X}}\right) \rightarrow \mathrm{H}^{0}\left(\mathbb{C}, \mathrm{Y} ; \mathrm{O}_{\mathrm{Y}}\right) \\
& \rightarrow \mathrm{H}^{1}\left(\mathbb{C}[t], \mathrm{X} ; \mathrm{O}_{\mathrm{X}}\right) \stackrel{\bullet t}{\rightarrow} \mathrm{H}^{1}\left(\mathbb{C}[t], \mathrm{X} ; \mathrm{O}_{\mathrm{X}}\right) \stackrel{\alpha}{\rightarrow} \mathrm{H}^{1}\left(\mathbb{C}, \mathrm{Y} ; \mathrm{O}_{\mathrm{Y}}\right) \\
& \rightarrow \mathrm{H}^{2}\left(\mathbb{C}[t], \mathrm{X} ; \mathrm{O}_{\mathrm{X}}\right) \stackrel{\bullet t}{\rightarrow} \mathrm{H}^{2}\left(\mathbb{C}[t], \mathrm{X} ; \mathrm{O}_{\mathrm{X}}\right) \rightarrow \cdots
\end{aligned}
$$

where $o_{X}$ is a $\mathbb{C}[t]$-algebra via $f^{\star}$. As in the proof of wahls conjecture $\operatorname{dim}_{\mathbb{C}}(\operatorname{Im}(\alpha))$ equals the dimension of the smoothing component on which $f: X \rightarrow \mathbb{C}$ "l:es", [G-L].

The algebra homomorphisms $\mathbb{C} \rightarrow \mathbb{C}[t] \stackrel{f^{\star}}{\rightarrow} \mathrm{O}_{X}$ induce the exact sequence:

$$
\begin{aligned}
\cdots & \rightarrow H^{1}\left(\mathbb{C}[t], X ; O_{X}\right) \rightarrow H^{1}\left(\mathbb{C}, X ; O_{X}\right) \rightarrow H^{1}\left(\mathbb{C}, \mathbb{C}[t] ; O_{X}\right) \\
& \rightarrow H^{2}\left(\mathbb{C}[t], X ; O_{X}\right) \rightarrow H^{2}\left(\mathbb{C}, X ; O_{X}\right) \rightarrow H^{2}\left(\mathbb{C}, \mathbb{C}[t] ; O_{X}\right) \rightarrow
\end{aligned}
$$

Now $H^{i}\left(\mathbb{C}, \mathbb{C}[t] ; O_{X}\right)=0$ for $i \geq 1$ so $H^{2}\left(\mathbb{C}[t], x ; O_{X}\right) \sim H^{2}\left(\mathbb{C}, X ; o_{X}\right)$. Since $f^{\star}(t)=f$ we get a short exact sequence

$$
O \rightarrow \operatorname{Im}(\alpha) \rightarrow \mathrm{T}_{Y}^{1} \rightarrow \mathrm{T}_{\mathrm{X}}^{2} \rightarrow 0
$$

proving the lemma.

Lemma 1.2. In the situation of 1.1 assume also that $x$ is a surface with $\mathbb{C}^{\star}$-action and $f$ is homogeneous under this action. Then

$$
\operatorname{dim}_{\mathbb{C}^{T}} \mathrm{~T}_{Y}^{1}-\mu(f)=\operatorname{dim}_{\mathbb{C}} \mathrm{T}_{X}^{2}+t_{Y}-1
$$

where $\mu(f)$ is the Milnor number of $f$ in $x$ and $t_{Y}$ is the Gorenstein type of $Y$.

Proof. In [Gr] it is proved that $e=\mu+t-1$ for quasi-homogeneous isolated curve singularities.

(As an obvious consequence of 1.2 , we see that if $\underline{m}_{X, x} \cdot T_{X}^{2}=0$, then $\tau-\mu$ for quasi-homogeneous functions on $X$ depends only on the surface, qeneralizing the fact that $\tau=\mu$ if $X$ is smooth.) 


\section{2. $T^{1}$ for rings over subsemigroups of free abelian semigroups.}

In this section $k$ denotes an algebraically closed field. In [La-S] Laudal and sletsjфe show that for a monoid algebra $k[\Lambda]$, the algebra cohomology groups $\mathrm{H}^{i}(\mathrm{k}, \mathrm{k}[\Lambda] ; \mathrm{M}),(\mathrm{M}$ a $\mathrm{k}[\Lambda]$-module), are isomorphic to the cohomology of the monoid with values in $M$. The algebra cohomology can then be computed using only the monoid structure. We shall use their ideas to find directly a method for computing $\mathrm{T}^{l}$ for monoid algebras $\mathrm{k}[\Lambda]$, when $\Lambda \subseteq \mathrm{z}_{0}^{\mathrm{n}} \cdot \mathrm{z}_{0}$ is the semigroup of non-negative integers.) The main examples are affine toric varieties.

Let $\Lambda$ be the submonoid of $z_{0}^{n}$ generated minimally by $v_{1}, \ldots, v_{m}$, and $k[\Lambda]$ the corresponding monoid algebra. Let $k\left[z_{0}^{n}\right]=k\left[t_{1}, \ldots, t_{n}\right]$. We may assume $n$ minimal so that $\operatorname{dim} k[\Lambda]=$ $\operatorname{rank} \Lambda=n$. Define $\rho: z_{0}^{m} \rightarrow \Lambda$ by $\rho\left(a_{1}, \ldots, a_{m}\right)=\sum_{i=1}^{m} a_{i} v_{i}$, and $\rho^{\star}: k\left[x_{1}, \ldots, x_{m}\right] \rightarrow k[\Lambda]$, by $x_{i} \rightarrow t^{v_{i}}$. Then $I=k e r \rho^{\star}$ is generated by $\left\{x^{c}-x^{d} \mid \rho(c)=\rho(d)\right\} \quad\left(x^{c}=x_{1}{ }^{c} 1 \ldots x_{m}{ }^{c}\right),[G]$, Thm.7.2. Extend $\rho$ to $\mathrm{z}^{\mathrm{m}} \rightarrow \mathrm{z}^{\mathrm{n}}$ and let $\mathrm{J}$ be the kernel. $\mathrm{J}$ is a free abelian group of rank $m-n=r=\operatorname{codim} k[\Lambda]$.

Lemma 2.1. Let $c, d_{1} a_{1}, \ldots, a_{r}, b_{1}, \ldots, b_{r}$ (not necessarily distinct) in $z_{0}^{m}$ be such that $c-d=\sum_{i=1}^{r}\left(a_{i}-b_{i}\right)$. There exist $\beta_{0}, \beta_{1}, \ldots, \beta_{r} \epsilon$ $\mathrm{z}_{0}^{\mathrm{m}}$ such that

$$
x^{\beta} 0\left(x^{c}-x^{d}\right)=\sum_{i=1}^{r} x^{\beta}\left(x^{a} i-x^{b}\right)
$$

Proof. The system of equations $\beta_{0}+c=\beta_{1}+a_{1} \beta_{i-1}+b_{i-1}=$ $\beta_{i}+a_{i}, i=2, \ldots, r$ has a solution in $z_{0}^{m}$. 
Let $\left\{j_{1}, \ldots, j_{r}\right\}$ be a basis for $J$ and let $\left(a_{i}, b_{i}\right)=$ $\left(\left(a_{i, 1}, \ldots, a_{i, m}\right),\left(b_{i, 1}, \ldots, b_{i, m}\right)\right)$ be the unique element in $z_{0 \Lambda}^{m} z_{0}^{m}$ such that $a_{i}-b_{i}=j_{i}$ and $a_{i, k} \cdot b_{i, k}=0$. Then $\left\{f_{i}=x^{a_{i}}-x^{b}\right\}_{i=1}^{r}$ is a maximal regular sequence for $k\left[x_{1}, \ldots, x_{m}\right]$ in $I$. In fact, one can use 3.1 to prove that $\operatorname{dim} k[\Lambda]=\operatorname{dim} k[\underline{x}] /\left(f_{1}, \ldots, f_{r}\right)$. Since no monomial in $k\left[x_{1}, \ldots, x_{m}\right]$ can be in $I, 3.1$ shows that the evaluation map

$$
\operatorname{Hom}_{k[\Lambda]}\left(I / I^{2}, k[\Lambda]\right) \rightarrow \underset{i=1}{\oplus} k[\Lambda]
$$

defined by $\phi \rightarrow\left(\psi\left(f_{1}\right), \ldots, \psi\left(f_{r}\right)\right)$ is injective. Working in the quotient field $k(\Lambda) \subset k\left(t_{1}, \ldots, t_{n}\right)$ we get the following description of the image.

Lemma 2.2. Hom $_{k}[\Lambda]^{\left(I / I^{2}, k[\Lambda]\right)}$ is isomorphic to

$$
\left\{\left(\psi_{1}, \ldots, \psi_{r}\right) \in \underset{i=1}{\oplus} k[\Lambda] \mid \sum_{i=1}^{r} \alpha_{i} t^{\rho(c)-\rho\left(a_{i}\right)} \cdot \psi_{i} \in k[\Lambda] \text {, for all } x^{c}-x^{d}\right.
$$

generating $I$ and $c-d=\sum_{i=1}^{r} \alpha_{i} j_{i}$ in $\left.J\right\}$.

Proof. From 3.1 there exist $\beta_{0}, \beta_{1}, \ldots, \beta_{r} \in \mathrm{z}_{0}^{\mathrm{m}}$ such that

$$
x^{\beta} 0\left(x^{c}-x^{d}\right)=\sum_{i=1}^{r} \operatorname{sign}\left(\alpha_{i}\right) \cdot x^{\beta}\left(x^{\left|\alpha_{i}\right| a_{i}} x^{\left|\alpha_{i}\right| b_{i}}\right) \text {. }
$$

Notice that

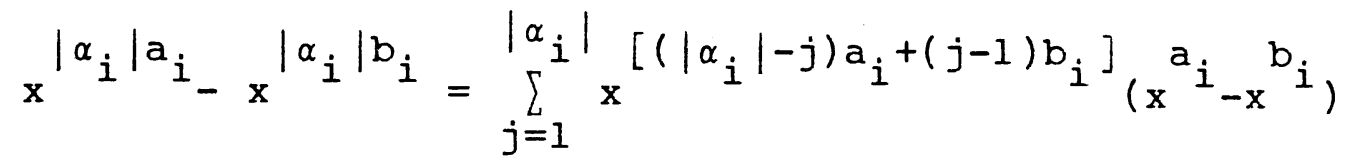

so if $\psi \in \operatorname{Hom}_{k[\Lambda]}\left(I / I^{2}, k[\Lambda]\right)=\operatorname{Hom}_{k[\underline{x}]}(I, k[\Lambda])$ then

$$
\psi\left(x^{\left|\alpha_{i}\right| a_{i}}\left|\alpha_{i}\right| b_{i}\right)=\sum_{j=1}^{\left|\alpha_{i}\right|} t^{\left(\left|\alpha_{i}\right|-1\right) \rho\left(a_{i}\right)}{ }_{\psi\left(x^{i}-x^{a}\right.} b
$$

Since $\rho\left(\beta_{i}\right)=\rho\left(\beta_{0}\right)+\rho(c)-\left|\alpha_{i}\right| \rho\left(a_{i}\right)$ we get

$$
\text { (*) } \psi\left(x^{c}-x^{d}\right)=\sum_{i=1}^{r} \alpha_{i} t^{\rho(c)-\rho\left(a_{i}\right)}{ }_{\psi\left(x^{i}-x^{b}\right) \in k[\Lambda]}
$$


On the other hand if $\left(\psi_{1}, \ldots, \psi_{r}\right)$ satisfy the conditions of 3.2 then using 3.1 and $(*)$ one checks that all relations among generators of I are satisfied.

Using this method one can e.g. compute a basis of $\mathrm{T}^{\mathrm{l}}$ for cyclic quotient singularities getting the equations of the first order deformations. The computation is similar to the one in $\$ 6$. See also $[R 1],[P 2],[\mathrm{La}-\mathrm{S}]$ and $[\mathrm{B} \cdot \mathrm{K}-\mathrm{R}]$ for other descriptions of $\mathrm{T}^{1}$ in this case. For a description of $\mathrm{T}^{1}$ for monomial curves see [Bu].

\section{Cyclic quotient singularities}

If $G$ is a finite cyclic subgroup of $G L(2, \mathbb{C})$, let $X=\mathbb{C}^{2} / G$ be the orbit space. It is a normal algebraic variety $\operatorname{Spec}\left(\mathbb{C}[\mathrm{x}, \mathrm{y}]^{\mathrm{G}}\right)$, where $\mathbb{C}[\mathrm{x}, \mathrm{y}]^{\mathrm{G}}$ is the invariant ring of the induced action. Since the origin is the only fixed point for the action of $G$, the corresponding point in $\mathrm{X}$ is an isolated singularity, the cyclic quotient singularity.

We may assume that $G$ contains no pseudo-reflections and, since $G$ is abelian, that $G$ is generated by the linear transformation

$$
\left(\begin{array}{cc}
\zeta_{n}^{q} & 0 \\
0 & \zeta_{n}
\end{array}\right)
$$

where $\zeta_{n}$ is a primitive $n '$ th root of unity, $n=0$ dG, and $0<q<n$, $\operatorname{gcd}(n, q)=1$. G's induced action on $\mathbb{C}[x, y]$ is generated by $x \rightarrow \zeta_{n}^{m}, y \rightarrow \zeta_{n}^{-1} y$ where $m=n-q$.

If $\Lambda \subset \mathrm{z}_{0}^{2}$ is the semigroup

$$
\Lambda=\left\{(\alpha, \beta) \in \mathrm{z}_{0}^{2} \mid \beta \equiv m \cdot \alpha(\mathrm{n})\right\}
$$


then $\mathbb{C}[x, y]^{\mathbf{G}}$ is the semigroup ring $\mathbb{C}[\Lambda]$. On $\Lambda$ we have the natural partial order: $\lambda_{1}>\lambda_{2}$ if there exists $\mu \in \Lambda$ such that $\lambda_{2}+\mu=\lambda_{1}$. This is just the restriction of the natural partial order on $\mathrm{z}_{0}^{2}$.

Let $\left\{v_{0}, \ldots, v_{r+1}\right\}$ be the minimal elements of $\Lambda\{0\}$ in this order. Write $v_{i}=\left(a_{i}, b_{i}\right)$ and order the indices so that $a_{i+1}>a_{i}, b_{i+1}<b_{i}$. Then for each $i=1, \ldots, r$ there is a number $e_{i}>2$ such that $v_{i-1}+v_{i+1}=e_{i} v_{i}$. These numbers appear in the continued fraction expansion

$$
\begin{array}{r}
\frac{n}{m}=e_{1}-\frac{1}{e_{2}-\frac{1}{e_{3}}} \\
\bullet-\frac{1}{e_{r}}
\end{array}
$$

and we could also define $v_{i}$ by $a_{0}=0, a_{1}=1$, $a_{i+1}=e_{i} a_{i}-a_{i-1}, b_{0}=n, b_{1}=m, b_{i+1}=e_{i} b_{i}-b_{i-1}$.

The minimal embeddings dimension is therefore $r+2$. If $\mathbb{C}\left[z_{0}, \ldots, z_{r+1}\right] \rightarrow \mathbb{C}[\Lambda]$ is the map $z_{i} \rightarrow x^{a_{i}}{ }^{b_{i}}$ then the kernel is generated minimally by $\frac{1}{2} r(r+1)$ polynomials

$$
g_{i j}=z_{i} z_{j}-z_{i+1} z_{j-1} \prod_{k=i+1}^{j-1} z_{k} e_{k}-2
$$

for $1 \leqslant i+1 \leqslant j-1 \leqslant r .([R I])$.

From now on we assume $r \geqslant 2$.

4. The Milnor number of a function on a cyclic quotient.

For the definitions needed below see $[\mathrm{K}]$.

Lemma 4.1 . Let $(f, 0)$ be the germ of an analytic function $f$ on the cyclic quotient singularity $(x, 0), x=\mathbb{C}^{2} / G$ and $n=o r d G$. If 
$\pi: \mathbb{C}^{2} \rightarrow \mathrm{X}$ is the natural projection set $\overline{\mathrm{f}}=\mathrm{fo} \pi$. Assume $\overline{\mathrm{f}}$ is non-degenerate and "commode" in the sense of Kouchnirenko [K]. Choose s,t minimally such that $x^{s \cdot n}, y^{t \cdot n}$ appear as monomials in $\bar{f}$. If $A$ is the area bounded by the Newton polygon of $\bar{f}$ and $S=A / n$, then the Milnor number $\mu(f, 0)$ equals $2 s-s-t+1$.

Proof. Embed $x$ in $\mathbb{C}^{r+2}$ with $z_{i}=x^{a_{i}{ }^{b}}{ }$ as in $\$ 3$. Using weighted balls

$$
B_{\varepsilon, N}=\left\{\left.x \in \mathbb{C}^{r+2}\left|\sum_{i=0}^{r+1}\right| z_{i}\right|^{2 W_{i}}<\varepsilon, \varepsilon>0, w_{i} \cdot\left(a_{i}+b_{i}\right)=N\right\}
$$

one constructs good representatives ([Lo], chap.3) for $f$ and $\bar{f}$ with Milnor fiber $F$ and $\bar{F}$ such that $F \simeq \bar{F} / G$. Thus $\chi(\bar{F})=n \cdot \chi(F)$ and $\mu(f, 0)=1+\frac{\mu(\bar{f}, 0)-1}{n}$. From $[K], \mu(\bar{f}, 0)=$ $2 A-s n-t n+1$, so $\mu(f, 0)=2 s-s-t+1$. (One checks that since $\bar{f}$ is invariant, $A \equiv 0(n))$

The lemma can be generalized to invariant functions for abelian finite subgroups of $G L(d, \mathbb{C})$, see $[M]$. The assumption "commode" is not essential.

\section{Monomial curves on cyclic quotients.}

We will now apply 1.2 to the cyclic quotient singularities. We wish to find a hypersurface for which the invariants are easily computed.

Proposition 5.1. With the notation of $\$ 3$ let $p$ be a positive integer such that $\operatorname{gcd}(p+m, n)=1$. Then $f=z_{0}^{P}-z_{r+1} \in \mathbb{C}[\Lambda]$ satisfies the conditions of 1.2 and $C=\operatorname{Spec}(\mathbb{C}[\Lambda] /(f))$, is a monomial curve (i.e. $\mathbb{C}[\Lambda] /(f) \simeq \mathbb{C}[\Gamma]$ for a semigroup $\Gamma \subset \mathrm{z}_{0}$ ). 
The proposition will follow from 5.2 and 5.3, but first a closer look at $T_{X}^{2}$. If $P=\mathbb{C}\left[z_{0}, \ldots, z_{r+1}\right]$ and $I=k e r(P \rightarrow \mathbb{C}[\Lambda])$, then the relation module $R=R(I)$ is the kernel of the $P$-homomorphism $\mathrm{P}^{\frac{1}{2} r(r+1)} \rightarrow I, E_{i j} \rightarrow g_{i j}$, where $E_{i j}$ is the standard basis of $\mathrm{P}^{\frac{1}{2} r(r+1)}, 1<i+1<j-1<r$. Let $\mathrm{R}_{0} \subset \mathrm{R}$ be the submodule generated by the trivial relations $g_{\alpha, \beta} \cdot g_{i j}-g_{i j} \cdot g_{\alpha, \beta}=0$. Recall that

$$
\mathrm{T}_{\mathrm{X}}^{2}=\mathrm{H}^{2}(\mathbb{C}, \mathrm{X} ; \mathbb{C}[\Lambda]) \simeq \operatorname{Hom}_{\mathrm{P}}\left(\mathrm{R} / \mathrm{R}_{0}, \mathbb{C}[\Lambda]\right) / \text { Der }
$$

where Der is generated by the derivations

$$
D_{\underline{h}}\left(R_{1}, \ldots, R_{\frac{1}{2}} r(r+1)\right)=\sum_{i=1}^{\frac{1}{2} r(r+1)} h_{i} R_{i}
$$

$\underline{h} \in \mathbb{C}[\Lambda]^{\frac{1}{2} r(r+1)},([L i-S],[L])$. In our case $R$ is generated by

$$
R_{i, j, k}=z_{i} E_{j k}-z_{j} E_{i k}+z_{k-1} \prod_{\ell=j+1}^{k-1} z_{\ell}{ }_{\ell}^{-2} E_{i, j+1}
$$

and

$$
s_{i, j, k}=z_{i+1} \prod_{\ell=i+1}^{j} z_{\ell}{ }^{e^{-2}} E_{j k}-z_{j+1} E_{i k}+z_{k} E_{i, j+1}
$$

for $0<i<j<k-1<r .([R I])$.

Lemma 5.2. $\quad\left(\mathrm{z}_{0}, \mathrm{z}_{\mathrm{r}+1}\right) \subset \operatorname{Ann}\left(\mathrm{T}_{\mathrm{x}}^{2}\right)$

Proof. The relations among relations:

$$
z_{j+1} R_{i, j, k}-z_{j} S_{i, j, k} \in R_{0}
$$

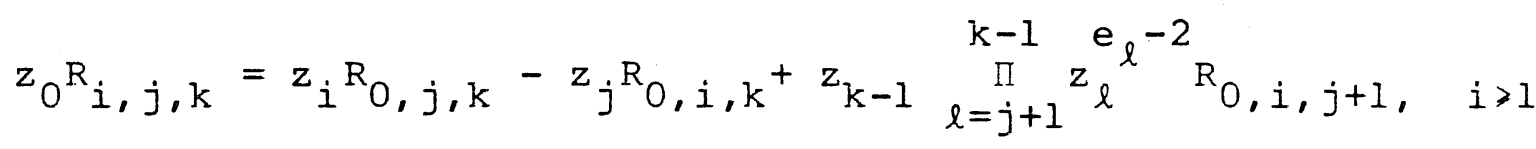

$z_{r+1} s_{i, j, k}=z_{i+1} \prod_{\ell=j+1}^{j} z_{\ell}^{e_{\ell}-2} s_{j, k-1, r+1}{ }^{-z} j+1 s_{i, k-1}, r+1+z_{k} s_{i, j, r+1}, k \leqslant r$ make $\phi_{1}, \phi_{2}: \operatorname{Hom}_{\mathrm{P}}\left({ }^{\mathrm{R}} / \mathrm{R}_{0}, \mathbb{C}[\Lambda]\right) \rightarrow \mathbb{C}[\Lambda]^{\frac{1}{2}(r-1) r}$

$$
\begin{aligned}
& \phi_{1}(\psi)=\left(\psi\left(R_{0,1,3}\right), \ldots, \psi\left(R_{0, j, k}\right), \ldots, \psi\left(R_{0, r-1, r+1}\right)\right) \\
& \phi_{1}(\psi)=\left(\psi\left(s_{0,1, r+1}\right), \ldots, \psi\left(s_{i, j, r+1}\right), \ldots, \psi\left(s_{r-2, r-1, r+1}\right)\right)
\end{aligned}
$$

injective. Let 


$$
\delta_{1}=\left(\frac{\overline{\partial R_{0, j, k}}}{\partial E_{\alpha, \beta}}\right) \quad \begin{aligned}
& 1<j<k-1<r \\
& 1<\alpha+1<\beta-1<r
\end{aligned}
$$

and

$$
\delta_{2}=\left(\frac{\overline{\partial S_{i, j, r+1}}}{\partial E_{\alpha, \beta}}\right) \quad \begin{aligned}
& 0<i<j<r-1 \\
& 1<\alpha+1<\beta-1<r
\end{aligned}
$$

(If $\mathrm{p} \in \mathrm{P}$, then $\overline{\mathrm{p}}$ is the image in $\mathbb{C}[\Lambda]$ ). $\mathrm{T}_{\mathrm{X}}^{2}$ is injectively mapped into $\mathbb{C}[\Lambda]^{\frac{1}{2}(r-1) r} / \mathrm{im \delta}_{1}$ and $\mathbb{C}[\Lambda]^{\frac{1}{2}(r-1) \mathrm{r} / \mathrm{im} \delta_{2}}$ by $\phi_{1}$ and $\phi_{2}$. For $\alpha \geqslant 1, z_{0}=\frac{\partial R_{0, \alpha, \beta}}{\partial E_{\alpha, \beta}}$ and $\frac{\partial R_{0, j, k}}{\partial E_{\alpha, \beta}}=0$ for $(j, k) \neq(\alpha, \beta)$. The $\frac{1}{2}(r-1) r$ vectors $\left(z_{0}, 0, \ldots, 0\right),\left(0, z_{0}, 0, \ldots, 0\right), \ldots,\left(0, \ldots, 0, z_{0}\right)$ are thus in im $\delta_{1}$. Similarily the vectors $\left(z_{r+1}, 0, \ldots, 0\right), \ldots,\left(0, \ldots, 0, z_{r+1}\right)$ are in im $\delta_{2}$, proving the lemma.

Lemma 5.3. If $\rho: \Lambda \rightarrow \mathrm{z}_{0}$ is the semigroup homomorphism $\rho\left(\lambda_{1}, \lambda_{2}\right)=p \lambda_{1}+\lambda_{2}, p$ is a positive integer with $\operatorname{gcd}(p+m, n)=1$ and $\operatorname{im}(\rho)=\Gamma$, then the kernel of $\rho^{\star}: \mathbb{C}[\Lambda] \rightarrow \mathbb{C}[\Gamma]$ is generated by $z_{0}^{P}-z_{r+1}$.

Proof. We know that kerp ${ }^{\star}$, is generated by $\left\{x^{\lambda}-x^{\mu} \in \mathbb{C}[\Lambda] \mid \rho(\lambda)=\rho(\mu)\right\}$ ([Gi] Thm.7.2). Viewing $\Lambda$ as a subset of $\mathbb{R}^{2}$, let $[\lambda, \mu]$ be the line segment between $\lambda$ and $\mu$. We must show that if the slope of $[\lambda, \mu]$ is $-p$ then there is a $g \in \mathbb{C}[\Lambda]$ such that

$$
g \cdot\left(x^{(n, 0)}-x^{(0, n p)}\right)=x^{\lambda}-x^{\mu}
$$

We may assume that $[\lambda, \mu] \cap \Lambda=\{\lambda, \mu\}$. Write $\lambda=\left(\lambda_{1}, \lambda_{2}\right)$, $\mu=\left(\mu_{1}, \mu_{2}\right)$. Then $\mu_{2}-\lambda_{2}=p\left(\lambda_{1}-\mu_{1}\right)$ so $\left(\lambda_{1}-\mu_{1}\right) \cdot(p+m) \equiv 0(n)$. From the assumption $\lambda_{1}-\mu_{1} \equiv O(n)$, so $\mu_{2}-\lambda_{2} \equiv O(n)$. The lemma is now easily proven. 


\section{The invariants of $c[r]$.}

Let $C=\operatorname{spec} \mathbb{C}[\Gamma]$ be the curve in 5.1 and choose

$$
\mathrm{p}=\mathrm{n}-\mathrm{m}+1=\mathrm{q}+1
$$

From 4.1 the Milnor number is

$$
\mu(C, 0)=n p-p
$$

Since $X$ is a rational singularity and $C$ is a hypersurface in $x$,

$$
t_{X}=t_{C}=r
$$

$([\mathrm{W}])$. Whats left in formula 1.2 is ${ }^{\tau_{C}}$.

Proposition 6.1. If $C$ is the curve of 5.1 and $p=q+1$ then

$$
\operatorname{dim}_{\mathbb{C}^{T}}{ }_{C}^{1}=n p-p+r-1+r(r-2)
$$

Before the proof we must look closer at the semigroup $\Gamma$.

Lemma 6.2. (i) If $N \in \mathrm{Z}_{0}$, write $\mathrm{N}=\mathrm{s}$ ttn with $0 \leqslant s<\mathrm{n}, t \geqslant 0$. Then $N \in \Gamma$ iff $t n>$ qs

(ii) If $w_{i}=\rho\left(v_{i}\right), i=0, \ldots, r+l \cdot\left(i \cdot e \cdot w_{r+1}=p w_{0}\right)$, then $\left\{w_{0}, \ldots, w_{r}\right\}$ is a minimal generator set for $\Gamma$.

(iii) We have $w_{0}=n, w_{1}=n+l$ and $w_{i+1}=e_{i} w_{i}-w_{i-1}$ for $i=1, \ldots, r$.

The proof is left to the reader.

Lemma 6.3. (i) The kernel $J$ of the group homomorphism $w: z^{r+1} \rightarrow z, w\left(s_{0}, \ldots, s_{r}\right)=\sum_{i=1}^{r} s_{i} w_{i}$ is generated by

$$
j_{i}=-\varepsilon_{i-1}+e_{i} \varepsilon_{i}-\varepsilon_{i+1}, i=1, \ldots, r-1
$$

and

$$
j_{r}=-\varepsilon_{r-1}+e_{r} \varepsilon_{r}-p \varepsilon_{0}
$$


where $\left\{\varepsilon_{i}\right\}_{i=0}^{r}$ is the standard basis of $z^{r+1}$

(ii) The kernel I of the $\mathbb{C}$-algebra homomorphism $w^{\star}: \mathbb{C}\left[z_{0}, \ldots, z_{r}\right] \rightarrow \mathbb{C}[\Gamma], z_{i} \rightarrow t^{w_{i}}$ is minimally generated by

$$
g_{i j}=z_{i} z_{j}-z_{i+1} z_{j-1} \prod_{k=i+1}^{j-1} z_{k} e^{-2}, \quad 1<i+1<j-1<r-1
$$

and

$$
g_{i, r+1}=z_{i} z_{0}^{p}-z_{i+1} z_{r} \prod_{i+1}^{r} z_{k}^{e} k^{-2}, i=0, \ldots, r-1
$$

The weights of $g_{i j}$ are $w\left(g_{i j}\right)=w_{i}+w_{j}$

Lemma 6.4. Let $e_{1}, \ldots, e_{r}$ be positive integers, $e_{i} \geqslant 2$. Consider the system of $r$ equations

$$
\begin{aligned}
e_{1} x_{1}-x_{2} & =y_{1} \\
-x_{1}+e_{2} x_{2}-x_{3} & =y_{2} \\
-x_{i-1}+e_{i} x_{i}-x_{i+1} & =y_{i} \\
-x_{r-1}+e_{r} x_{r} & =y_{r}
\end{aligned}
$$

in $2 r$ variables $x_{i}$ and $y_{i}$. Let

$$
\begin{array}{r}
\frac{n}{m}=e_{1}-\frac{1}{e_{2}-\frac{1}{e_{3}}} \\
\ddots \ddots-\frac{1}{e_{r}}
\end{array}
$$

Define $a_{0}=0, a_{1}=1, a_{i+1}=e_{i} a_{i}-a_{i-1}$ and $b_{0}=n, b_{1}=m$ $b_{i+1}=e_{i} b_{i}-b_{i-1}$. Then

$$
x_{i}=\frac{1}{n}\left[b_{i}\left(\sum_{k=1}^{i} a_{k} y_{k}\right)+a_{i}\left(\sum_{k=i+1}^{r} b_{k} y_{k}\right)\right]
$$

Proof. The proof is easy when one notices that

$$
\begin{array}{r}
\frac{a_{i+1}}{a_{i}}=e_{i}-\frac{1}{e_{i-1}} \\
\ddots-\frac{1}{e_{1}}
\end{array}
$$

and that $a_{i+1} b_{i}-a_{i} b_{i+1}=n$ for $a l l$. $i=0, \ldots, r$. 
Proof of 6.3 . (i) If $\sum_{k=0}^{r} s_{i} w_{i}=0$ use 6.4 to solve $\left(s_{0}, \ldots, s_{r}\right)$ $=\sum_{k=1}^{r} \alpha_{r}{ }_{r}$ for $\alpha_{i} \in z . \quad(i i)$ is obvious from $\$ 3$.

We can now apply 2.2. The generator $g_{i j}$ of $I$ corresponds to the element

$$
\varepsilon_{i}+\varepsilon_{j}-\varepsilon_{i+1}-\varepsilon_{j-1}-\sum_{k=i+1}^{j-1}\left(e_{k}-2\right) \varepsilon_{k}=\sum_{k=i+1}^{j-1} j_{k}
$$

in J. So

$$
\begin{aligned}
& \operatorname{Hom}_{P}(I, \mathbb{C}[\Gamma]) \simeq\left\{\left(\phi_{1}, \ldots, \phi_{r}\right) \in \underset{i=1}{\oplus} \mathbb{C}[\Gamma] \mid\right. \\
& \sum_{\alpha=i+1}^{j-1} t^{w_{i}+w_{j}-e_{\alpha} w_{\alpha}} \cdot \phi_{\alpha} \in \mathbb{C}[\Gamma] \text {, for all } i, j \text { such that } \\
& 1<i+1<j-1<r\}
\end{aligned}
$$

One checks that the above criterion splits to each summand, i.e.

$$
\operatorname{Hom}_{P}(I, \mathbb{C}[\Gamma]) \simeq \underset{\alpha=1}{\oplus} \boldsymbol{\sigma}_{\alpha}
$$

where $\boldsymbol{\sigma}_{\alpha}=\left\{\phi \in C[\Gamma] \mid t^{w_{i}+w^{-e}} j^{-w_{\alpha}} \cdot \phi \in \mathbb{C}[\Gamma]\right.$ for all $i, j$ such that $i+1<\alpha \leqslant j-1\}=\bigcap_{i+1 \leqslant \alpha \leqslant j-1}\left(\left(t^{e^{\alpha} w_{\alpha}}\right):\left(t^{w_{i}+w_{j}}\right)\right)$

We now prove 6.1, omitting computational details, but giving necessary stepping stones as lemmas.

Lemma 6.5. (i) If $i \leqslant i+1 \leqslant j-1 \leqslant r$ then $w_{i}+w_{j}-\left(e_{\alpha}-1\right) w_{\alpha} \in \Gamma$ for $\alpha=i+1, \ldots, j-1$ and $w_{i}+w_{j}-w_{\alpha} \in \Gamma$ for $\alpha=i, \ldots, j$.

(ii) $\mathrm{pw}_{0}+\mathrm{w}_{i}-\left(e_{\alpha}-1\right) \mathrm{w}_{\alpha} \in \Gamma$ for $\alpha \in\{1, \ldots, r\}-\{i\}$ and $\mathrm{pw}_{0}+\mathrm{w}_{i}-\mathrm{w}_{\alpha} \in \Gamma$ for all $\alpha=0, \ldots, \mathrm{r}+1$.

(iii) If $h=0, \ldots, p$ and $\gamma \in \Gamma$ then $h \cdot w_{0}-\gamma \in \Gamma$ iff $\gamma=k \cdot w_{0}$ for a $k=0, \ldots, h$. 
Proof. (i) Continued use of the fact that $w_{i}+w_{j}=$ $w_{i+1}+w_{j-1}+\sum_{k=i+1}^{j+1}\left(e_{k}-2\right) w_{k} \cdot$ (ii) follows from (i) since $p_{0}=$ $(p-1) w_{0}+w_{0}=w_{r+1}$. For (iii) use 6.2 .

Using 6.5 one can prove

Lemma 6.6. $q_{\alpha}$ is the ideal generated by

$$
\begin{aligned}
& \left\{t^{w} \mid w \in\left\langle w_{\alpha-1}, w_{\alpha}, w_{\alpha+1}>\cup\left\{\mathrm{pw}_{0}+w_{\alpha+1}-w_{i} \mid\right.\right.\right. \\
& \left.i=\alpha+1, \ldots, r\} \cup\left\{\mathrm{pw}_{0}+w_{\alpha-1}-w_{i} \mid i=1, \ldots, \alpha-1\right\}\right\} .
\end{aligned}
$$

(Notation: $\left\langle\gamma_{1}, \ldots, \gamma_{k}\right\rangle$ is the semigroup ideal generated by $\left.\left\{\gamma_{1}, \ldots, \gamma_{k}\right\}\right)$.

Let $\delta$ be the matrix $\left(\frac{\overline{\partial g_{i-1, i+1}}}{\partial z_{\alpha}}\right)=$

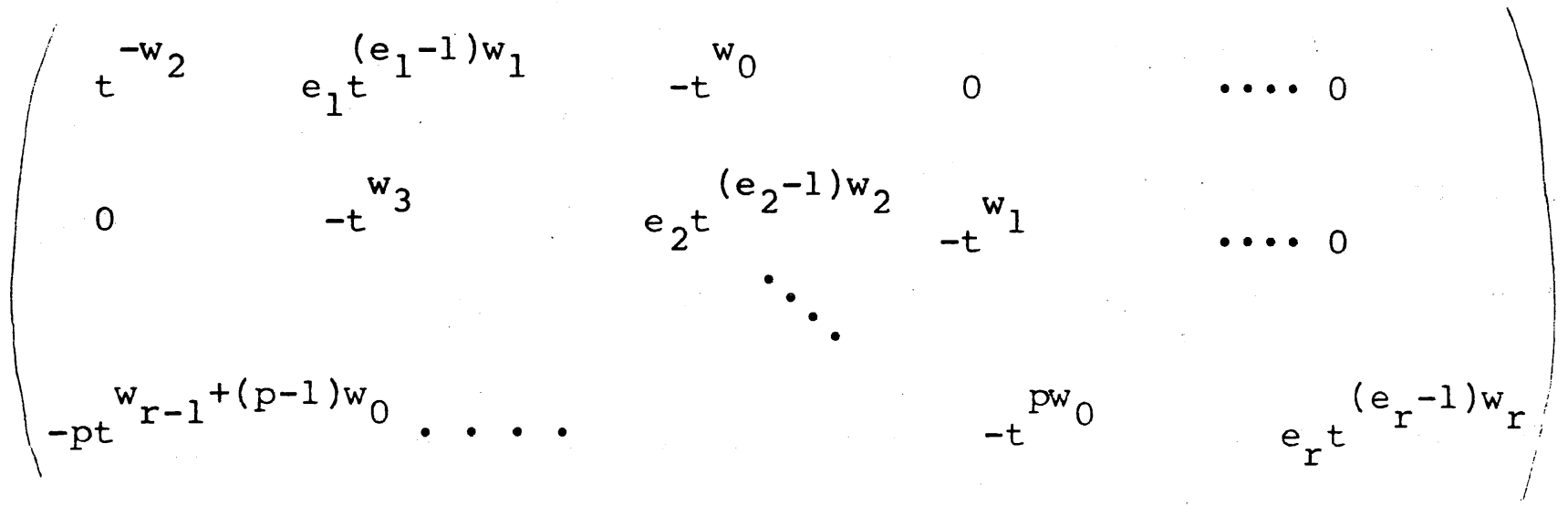

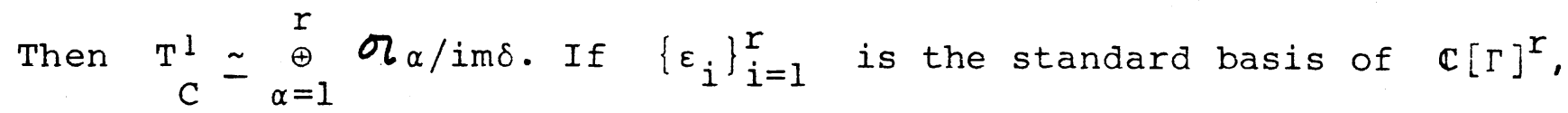
then a typical element in imo looks like

$$
\begin{aligned}
& \sum_{i=1}^{r-1}\left(-\phi_{i-1} \cdot t^{w_{i+1}}+e_{i} \phi_{i} t^{\left(e_{i}-1\right) w_{i}}-\phi_{i+1} \cdot t^{w_{i-1}}\right) \cdot \varepsilon_{i} \\
+ & \left(-\phi_{r-1} t^{p w_{0}}+e_{r} \phi_{r}{ }^{\left(e_{r-1}\right) w_{r}} r^{p \phi_{0}} t^{w_{r-1}+(p-1) w_{0}}\right) \cdot \varepsilon_{r}
\end{aligned}
$$

where $\phi_{0}, \ldots, \phi_{\Gamma} \in \mathbb{C}[\Gamma]$. A computation using $6.4,6.5,6.6$ and $(\star)$ gives 
Lemma 6.7. A basis for $T_{C}^{1} \simeq \oplus^{\gamma_{\alpha}} /$ im $\delta$ is

( I )

$$
\begin{aligned}
& \left\{t^{p w_{0}+w_{\alpha+1}-w_{i}} \cdot \varepsilon_{\alpha} \mid \alpha=2, \ldots, r-1, i=\alpha+1, \ldots, r\right\} U \\
& \left\{t^{p w_{0}+w_{\alpha+1}-w_{i}} \cdot \varepsilon_{\alpha} \mid \alpha=2, \ldots, r, i=1, \ldots, \alpha-2\right\} U \\
& \left\{t^{p w_{0}+w_{2}-w_{i}} \cdot \varepsilon_{1} \mid i=3, \ldots, r\right\} U
\end{aligned}
$$

(II) $\quad\left\{t^{k_{\alpha} \cdot w_{\alpha}} \cdot \varepsilon_{\alpha} \mid \alpha=1, \ldots, r, k_{\alpha}=1, \ldots, e_{\alpha-2}\right\} U$

$$
\begin{aligned}
& \left\{t^{w-1} \cdot \varepsilon_{\alpha} \mid \alpha=1, \ldots, r-1\right\} U \\
& \left\{t^{w_{\alpha+1}} \cdot \varepsilon_{\alpha} \mid \alpha=2, \ldots, r\right\} U
\end{aligned}
$$

(III) $\left\{t^{w_{r-1}+w} \cdot \varepsilon_{r} \mid w \in \Gamma-\left(\left\langle\mathrm{pw}_{0} \cdot(\mathrm{p}+1) \mathrm{w}_{0}-\mathrm{w}_{1}\right\rangle\right.\right.$

$$
\left.\left.U\left\{(p-1) w_{0}+(p+1) w_{0}-\left(e_{i}-1\right) w_{i} \mid i=1, \ldots, r\right\}\right)\right\}
$$

Proof of 6.1. The basis elements of type I and II sum up to $(r+1)(r-2)+\sum_{i=1}^{r}\left(e_{i}-1\right)$. To count the ones of type III notice the $1-1$ correspondance between $\Gamma-\left\langle\mathrm{pw}_{0}\right\rangle$ and $\left\{\left(\lambda_{1}, \lambda_{2}\right) \in \Lambda \mid \lambda_{1}<\mathrm{n}, \lambda_{2}<\mathrm{pn}\right\}$.

Remark. The basis elements of type III are first order deformations of $C$ in $X$, see $[C]$.

Adding up the invariants we get:

Theorem. If $r$ is the (minimal) codimension of the cyclic quotient singularity $X$ and $r \geqslant 2$ then

$$
\operatorname{dim}_{\mathbb{C}^{T}} T_{X}^{2}=r \cdot(r-2)
$$

Example. Let $\mathrm{X}$ be the affine cone over the embedding of $\mathbb{P}^{1}$ in $\mathbb{P}^{n}$ by $O_{\mathbb{P}^{l}}(n)$. Then $\mathrm{X}$ is the cyclic quotient with ordG=n and $q=1$. We have $\operatorname{dim} T_{X}^{1}=2 n-4$ and for $n>5$ the formal moduli space 
$S$ is geometrically smooth of codimension $n-1$ ([P2]). Since in this case $r=n-1$.

$$
\operatorname{dim} T_{X}^{2}=(n-1)(n-3)=(n-1)\left(\operatorname{dim} T_{X}^{1}-\operatorname{dim} S\right)
$$

In general for cyclic quotients $\operatorname{dim} \mathrm{T}^{1}=\left(\sum_{i=1}^{r} e_{i}\right)-2$, and there exists an Artin component $A$ of dimension $\sum_{i=1}^{r}\left(e_{i}-1\right)([R I])$ so

$$
\operatorname{dim} \mathrm{T}_{X}^{2}=r\left(\operatorname{dim} \mathrm{T}_{\mathrm{X}}^{1}-\operatorname{dim} \mathrm{A}\right)
$$

\section{References}

[B-K-R] K. Behnke, C. Kahn, O. Riemenschneider: "In finitesmal deformations of quotient surface singularities", Preprint Hamburg

[Br] E. Brieskorn: "Rationale Singularitäten Komplexer Flächen", Invent.Math. 4, 336-358 (1967-68)

[Bu] R.-O. Buchweitz: "On deformations of monomial curves", in Seminaire sur les Singularities des surfaces, Palaiseau 7677. Springer lecture Notes.

[C] J.A. Christophersen: "Derivations of simplicial conic algebras.", Preprint no 4 1985, Univ. of Oslo.

[Gi] R. Gilmer: Commutative semigroup rings, Chigago 1984

[Gr] G.-M. Greuel: "On deformations of curves and a formula of Deligne." in Algebraic Geometry, Proceedings, La Rabida, 1981, S.L.N. 961.

[G-L] G.-M. Greuel, E. Looijenga; "The dimension of smoothing components", Duke Math.J., 52, 263-272 (1985)

[K-K-M-S] G. Kempf, F. Knudsen, D. Mumford, B. Saint-Donat: Toroidal Embeddings, Springer Lecture Notes 339

[K] A.G. Kouchnirenko: "Polyhedra de Newton et nombres de Milnor". Inv.math. 32, 1-32 (1976).

[L] O.A. Laudal: Formal Moduli of Algebraic Structures, Springer Lecture Notes.

[L-P] O.A. Laudal, G. Pfister: The Local Moduli Problem. Applications to Isolated Hypersurface Singularities to appear. 
ŁLa-S」 O.A. Laudal, A. Sletsj申e: "Cohomology of groups, monoids and their algebras", manuscript.

[Li-S] S. Lichtenbaum and M. Schlessinger. "The cotangent complex of a morphism", Trans. A.M.S. 128, 41-70 (1967).

[Lo] E.J.N. Looi jenga: Isolated Singular Points on Complete Intersections, Cambridge 1984.

[M] M.A. Muffett: "Invariant Milnor numbers and Newton polyhedra." Thesis, Univ. of Liverpool.

[Pl] H.C. Pinkham. "Deformatons of algebraic varieties with $G_{m}$ action.", astérisque 20 (1974)

[P2] H.C. Pinkham: "Deformatons of quotient surface singularities", Proc. of symp. in Pure Math. 30, 65-67 (1977).

[R] O. Riemanschneider: "Deformationen von Quotienten singularitäten.", Math.Ann. 209 211-248 (1974)

[Ri] D.S. Rim, "Formal deformation theory", SGA 7(I), Exp VI

[S] M. Schlessinger: "Functors of Artin rings" Trans. A.M.S. 130 (1968)

[W] J.M. Wahl: "Equations defining rational singularities", Ann.Scient. Ec.Norm.Sup., $4^{e}$ série, t.10, 231-264 (1977). 
